\title{
Microscopy and Spectroscopy of Catalysts and Energy Storage Materials
}

\author{
S. Prabhudev ${ }^{1}$, S. Stambula ${ }^{1}$, L. Chinchilla ${ }^{1}$, C. Chiang $^{1}$, M. Chatzidakis ${ }^{1}$, H. Liu ${ }^{1}$, D. Rossouw ${ }^{1}$, C. \\ Wiktor $^{1}$, M. Bugnet ${ }^{1,2}$ and G.A. Botton ${ }^{1}$ \\ ${ }^{1}$ Department of Materials Science and Engineering, McMaster University, Hamilton, ON, Canada \\ ${ }^{2}$ Now at CNRS-INSA Lyon, UMR 5510, Bât. B. Pascal, F-69621 Villeurbanne Cedex, France
}

Electron microscopy has always played an important role in the development and the understanding of new materials. In the last ten years there have been significant advancements in instrumentation, enabling improved studies of materials at the nanoscale. In the area of catalysts and energy storage materials, detailed microscopy of material structure, composition and bonding at the nanometer length scale are needed to optimize material properties and performance. Here we summarize recent examples of work related to the study of nano-alloy catalysts used in proton exchange membrane fuel cells and commercial Li ion battery materials, illustrating the crucial role of imaging and spectroscopy for the characterization of these materials.

An FEI Titan (80-300 Cubed) microscope fitted with an electron energy loss spectroscopy (EELS) capability (Quantum 966) was used for this work. Using this instrument and quantitative image analysis, we have studied the mechanism platinum $(\mathrm{Pt})$ cluster formation following atomic layer deposition on graphene nanosheets. We have also shown that, with electron energy loss near-edge structures, it is possible to detect the presence of single $\mathrm{N}$ dopant atoms at different atomic sites [1]. With high-angle annular dark-field scanning transmission electron microscopy (HAADF-STEM) and EELS, we have studied the evolution of alloy catalysts following in-situ and ex-situ annealing procedures. Starting with a disordered $\mathrm{PtFe}$ nanoparticle, we captured the ordering transformation, showing evidence of ordered $\mathrm{Pt}$ and Fe rich planes formation, and evidence of both $\mathrm{Pt}$ and Fe-rich shells over a Pt-Fe ordered core (Figure 1) [2]. We also showed that the Pt surface segregation induces local strain and atomic displacements [2] (Figure 2) that can be further correlated to the enhanced catalytic activity of the material, in addition to the enhanced durability shown previously [3,4]. Using in-situ heating, and taking advantage of fast acquisition capabilities with EELS, it has also been possible to study the alloying phenomena of AuPt nanoparticles showing evidence of full miscibility starting at $200^{\circ} \mathrm{C}$ (Figure 3 ), well below the thermodynamically expected temperature, due to the reduced scale of the system. At hightemperature, we have also detected the formation of unexpected ordered structures (Figure 4). Furthermore, we found that the annealing under reducing atmospheres leads to mostly phase separation and monolayer surface segregation [5]. In a related catalyst system, we have been able to study the evolution of catalysts and hybrid supports, following identical location TEM-STEM-EELS, visualizing the presence of single atoms dissolution, and even electron tomography techniques [6]. These experiments demonstrate the value of multiple approaches for the characterization of the structure of engineering materials, not only model systems.

Similar approaches have been used to study the structure of $\mathrm{LiNi}_{\mathrm{x}} \mathrm{Mn}_{\mathrm{y}} \mathrm{Co}_{1-\mathrm{x}-\mathrm{y}} \mathrm{O}_{2}$ (known as "NMC") and high-energy (Ni rich) NMC cathode materials. In this work, using a combination of HAADF STEM and EELS, together with multiple-linear least squares fitting, we have demonstrated the mechanisms of charge compensation, following electrochemical cycling and the presence of monolayer-like surface changes in the valence of transition metal ions. STEM imaging also revealed the presence of local heterogeneities in the $\mathrm{Li}$ and Transition Metal distribution, at the single atomic column level, following 
several charge-discharge cycles. Finally, the limitations for single atom spectroscopy for this particular system will also be discussed [7].

\section{References:}

[1] S. Stambula et al., Journal of Physical Chemistry C, 118, 3890-3900, (2014)

[2] S. Prabhudev et al., ChemCatChem, 7, 3655-3664, (2015)

[3] S. Prabhudev et al., ACS Nano 7, 6103-6110, (2013)

[4] M.C.Y. Chan et al., Nanoscale 4 (22), 7273-7279, (2012)

[5] S. Prabhudev, C. Chiang, M. Chatzidakis et al., In Communication

[6] L. Chinchilla-Reyes, D. Rossouw et al. In Preparation

[7] The authors are grateful to NSERC for supporting this research. The microscopy was carried out at the Canadian Centre for Electron Microscopy, a National facility supported by The Canada Foundation for Innovation, under the MSI program, NSERC and McMaster.
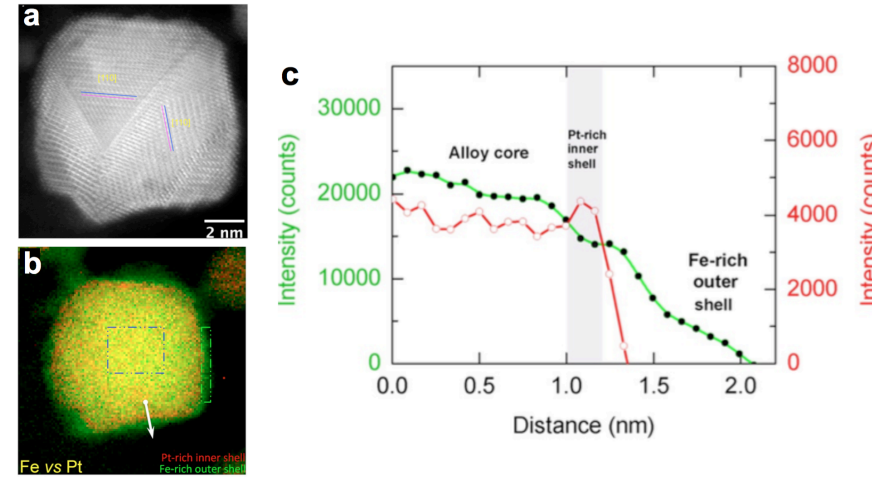

Figure 1: (a) and (b), STEM-HAADF and Pt versus Fe STEM-EELS composite map of a heat-treated Pt-Fe nanoparticle, respectively. (c) EELS line profiles of Fe L-edge (green) and Pt M-edge (red) taken along the white arrow highlighted in (b).


Figure. 3: STEM-HAADF image (1) and EELS chemical imaging ( 2,3 and 4$)$ of a thermally annealed Pt-Au nanoparticle. (2) and (3), 2D map of $\mathrm{Pt}$ and $\mathrm{Au}$, respectively. (4), composite Pt versus Au map. Clearly, these maps evidence a phase separation between $\mathrm{Pt}$ and $\mathrm{Au}$, corroborating with the immiscibility gap observed in the bulk phase diagram.

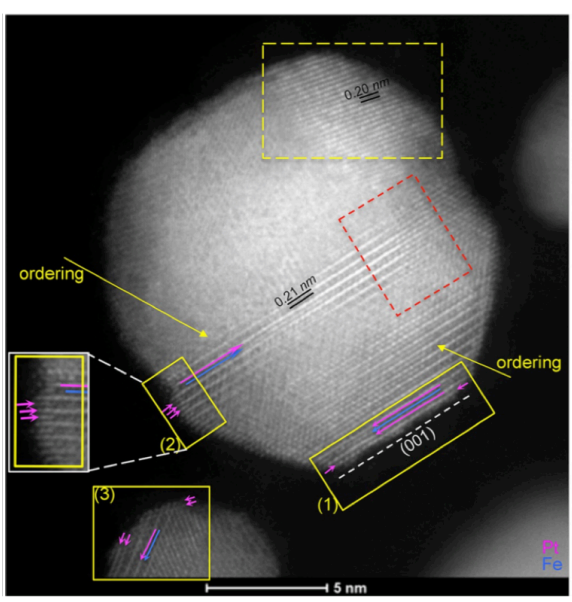

Figure 2: STEM-HAADF image of the ordered particle illustrating Pt atoms wedged at the surface (highlighted in the yellow boxes).

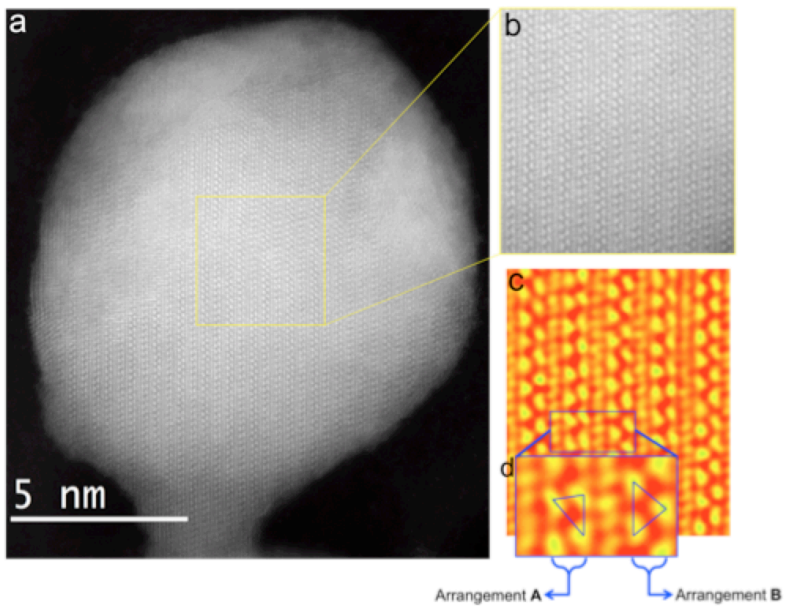

Figure 4: (a) STEM-HAADF image. (b and c) Enlarged images of the selected region in (a). 